
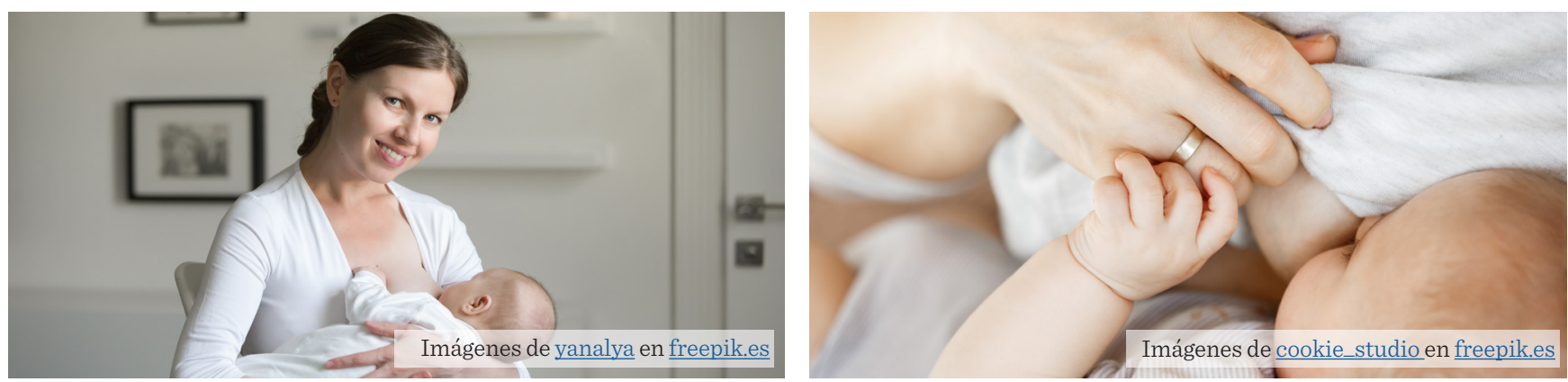

\title{
Conocimiento y actitudes sobre los beneficios de la lactancia materna en universitarios
}

\section{University students' knowledge and attitudes towards breastfeeding}

\author{
Juan Alejandro Hernández-Pérez', Miguel Ángel Hernández-Hernández', María de los Ángeles Ramírez-Trujillo*
}

\section{RESUMEN}

La lactancia materna (LM) tiene beneficios en la salud del binomio madre-hijo. El abandono y las barreras para realizar la práctica de amamantar se relacionan con cuestiones biológicas, ambientales, culturales, sociales e individuales, entre otras. Todas ellas tienen influencia del conocimiento y actitudes que se adquiere durante la vida. La población universitaria, es una generación de adultos que forman un criterio integral sobre LM en función de la dinámica social. El objetivo del presente trabajo fue analizar el nivel de conocimiento y las actitudes de los estudiantes universitarios respecto a los beneficios de la lactancia materna en la salud. Se observó bajo nivel de conocimiento entre la población universitaria sobre aspectos de importancia tales como, la duración de la LM exclusiva, la reducción del riesgo en el desarrollo de obesidad, infecciones de oído, diarrea o alergias alimentarias, especialmente en estudiantes que no fueron del área de la salud. Respecto a las actitudes, se identificó una alta aceptación social de la práctica de la LM. Sin embargo, los estudiantes consideran a la alimentación con fórmula como una opción más práctica. Es necesario realizar investigaciones que integren diversos sectores de la sociedad que permitan explicar los cambios en acciones y comportamientos hacia la LM.

\section{ABSTRACT}

Breastfeeding (BF) has health benefits for the mother-child dyad. Abandonment and barriers to breastfeeding are related to factors at different levels that include biological, environmental, cultural, social, individual issues, among others. All of them are shaped by the knowledge and attitude that are acquired during life. The university population is a generation of adults who develop a comprehensive criterion on BF based on social dynamics. The objective of the present work was to analyze the level of knowledge and attitudes that university students have regarding the health benefits of $\mathrm{BF}$. It was observed a low level of the knowledge about relevant aspects such as the duration of exclusive $\mathrm{BF}$, the risk reduction in the development of obesity, ear infections, diarrhea or food allergies. Regarding attitude, a high level of social acceptance of the BF practice was identified. However, formula feeding is considered a more practical option. It is necessary to carry out further research that integrates various sectors of society that allow explaining the changes in actions and behaviors towards $\mathrm{BF}$.

KEYWORDS: breastfeeding, students, benefits, knowledge.

PALABRAS CLAVE: amamantar, estudiantes, beneficios, conocimiento.

*Correspondencia: mramirez@enes.unam.mx/ Fecha de recepción: 8 de febrero de 2021/Fecha de aceptación: 22 de julio de 2021/Fecha de publicación: 2 'y de julio de 2021.

${ }^{1}$ Universidad Nacional Autónoma de México, Licenciatura en Odontología, Escuela Nacional de Estudios Superiores Unidad León, San Antonio de los Tepetates, León, Guanajuato, México. ${ }^{2}$ Universidad Nacional Autónoma de México, Departamento de Salud Pública, Escuela Nacional de Estudios Superiores Unidad León, boulevard UNAM núm. 2011, col. Predio El Saucillo y El Potrero, San Antonio de los Tepetates, León, Guanajuato, México, C. P. 37689. 


\section{INTRODUCCIÓN}

La práctica de la lactancia materna (LM) inicia a la par del origen del ser humano y desde la antigüedad ha sido un medio de supervivencia de los niños, acorde a la información de la Organización Mundial de la Salud (OMS, 1981). La leche materna modifica su composición de acuerdo con la edad del niño y su uso se sugiere hasta los 24 meses de vida o más, si la madre e hijo así lo desean (OMS, 1981; OMS, 2020).

Existe evidencia científica sólida sobre los múltiples beneficios de la LM en la salud social, emocional y física, tanto de la madre como del hijo. Influye a nivel social favoreciendo el apego, el vínculo madre e hijo, de acuerdo con la Asociación Española de Pediatría (AEPED, 2012); asimismo, la satisfacción emocional materna por el hecho de proveer las necesidades del bebé (Maslow, 1975). En la madre, la lactancia protege de enfermedades crónicas como hipertensión, obesidad, diabetes, depresión, incluso cáncer (Paredes-Juárez, 2019). La LM protege a los lactantes en sus primeros meses de vida de enfermedades como la diarrea, la neumonía, otitis media, enfermedades respiratorias, además de que favorece el desarrollo cognitivo y evita la muerte repentina e inexplicable en niños menores de un año (Mosca y Gianni, 2017; Paredes-Juárez, 2019). La LM salva vidas, mejora la salud de los niños que la consumen en el corto y largo plazo, además de potencializar su desarrollo cognoscitivo, mientras que las consecuencias inmediatas de la mal nutrición durante los primeros 3 años de vida incluyen un mayor riesgo de enfermar y morir, así como un desarrollo mental y motor retrasado (González y Ferré, 2016).

De acuerdo con la OMS, se estima que a nivel mundial 78 millones de recién nacidos no reciben lactancia materna exclusiva (LME) en la primera hora de vida, lo que predispone en la mayoría de los casos a no alimentar con leche materna. En promedio solo el $38 \%$ de los lactantes reciben LME en todo el mundo. Las tasas más altas de LM se han reportado en países de África Oriental (65 \%) y las más bajas en países de ingresos medios y altos como Asia Oriental y el Pacífico (32 \%) (OMS, 2020).

El abandono y las barreras para realizar la LM se deben a factores en diferentes niveles. El primer nivel se relaciona con características del bebé, de la madre y ambos como binomio, por ejemplo, influye el peso inicial del bebé, la incapacidad o el rechazo del amamantamiento, la salud materna, dolor al amamantar, falta de conocimiento en las madres sobre los beneficios de la lactancia, entre otros (Patil y col., 2020).

El conocimiento necesario para revalorar la práctica de la LM es el relativo a los beneficios que otorgan el calostro y la duración de la LME, traducidos en beneficios económicos, así como en la salud de la madre, ya que se asocia con una reducción del riesgo de cáncer de mama, de ovario, eventos cardiovasculares y depresión post-parto (González y Ferré, 2016).

Las actitudes ideales sobre la LM están enfocadas en reconocer la importancia de los beneficios nutricionales de la leche materna como alimento ideal para los bebés, la relevancia en el desarrollo del apego y la unión madre-hijo, así como en la aceptación y el respeto a la exhibición pública del amamantamiento (Heathman y col., 2019).

Diversas instituciones y organizaciones que buscan promover la LM a nivel internacional, sugieren realizar investigaciones que integren el análisis de sectores poblacionales diversos que permitan explicar los cambios en acciones o comportamientos hacia la LM a través del tiempo (González y Ferré, 2016). En este sentido, una población de interés para el estudio de factores que intervienen en la práctica de amamantar es la comunidad universitaria. La relevancia de identificar el conocimiento y actitudes sobre los beneficios de la LM en universitarios radica en que es una generación próxima para convertirse en padres o madres y/o prestadores de algún servicio en el área de la salud, es decir, una nueva generación de adultos con criterio sobre la LM en función de la diná- 
mica social. Por lo tanto, es importante identificar el nivel de conocimiento y la predisposición que se tenga respecto a la LM. Hasta el momento son pocos los estudios que realizan una revisión sobre el tema.

El objetivo de este trabajo fue analizar el nivel de conocimiento y actitudes que tienen los estudiantes universitarios respecto a los beneficios de la lactancia materna en la salud madre-hijo.

\section{Identificación de bibliografía}

La revisión bibliográfica se realizó con base a los lineamientos de la declaración PRISMA (Figura 1). En la estrategia metodológica PICO se incluyeron: $\mathrm{P}$ (población) = estudiantes universitarios, I (intervención) y C (comparación) = no aplicaron, y $\mathrm{O}$ (resultados) = conocimiento sobre los beneficios de la LM para la madre y el bebé, indicaciones para amamantar, vínculos afectivos, duración de la lactancia y com- prensión de la LM; actitudes sobre los beneficios de la LM, relaciones familiares y apego, beneficios de la LM, duración de la LM, uso de fórmula y biberón, trabajo y lactancia, exposición de la lactancia en público.

La búsqueda bibliográfica se llevó a cabo en octubre de 2020, por 3 personas de manera independiente en las bases de datos Medline, Scopus y PubMed, con palabras clave en ambos idiomas: amamantar-"breastfeeding"; conocimiento-“knowledge"; amamantar-"breastfeeding”; beneficios-"benefits"; amamatar-"breastfeeding"; salud-"health"; actitudes-"attitudes"; estudiantes-"students"; beneficios-"benefits".

Se analizaron aquellos artículos relacionados con el conocimiento y actitudes que tienen los universitarios respecto a los beneficios de la LM en la salud del binomio madre-hijo. Se seleccionaron los artículos que incluyeran estudios de corte transversal, longitudinales o

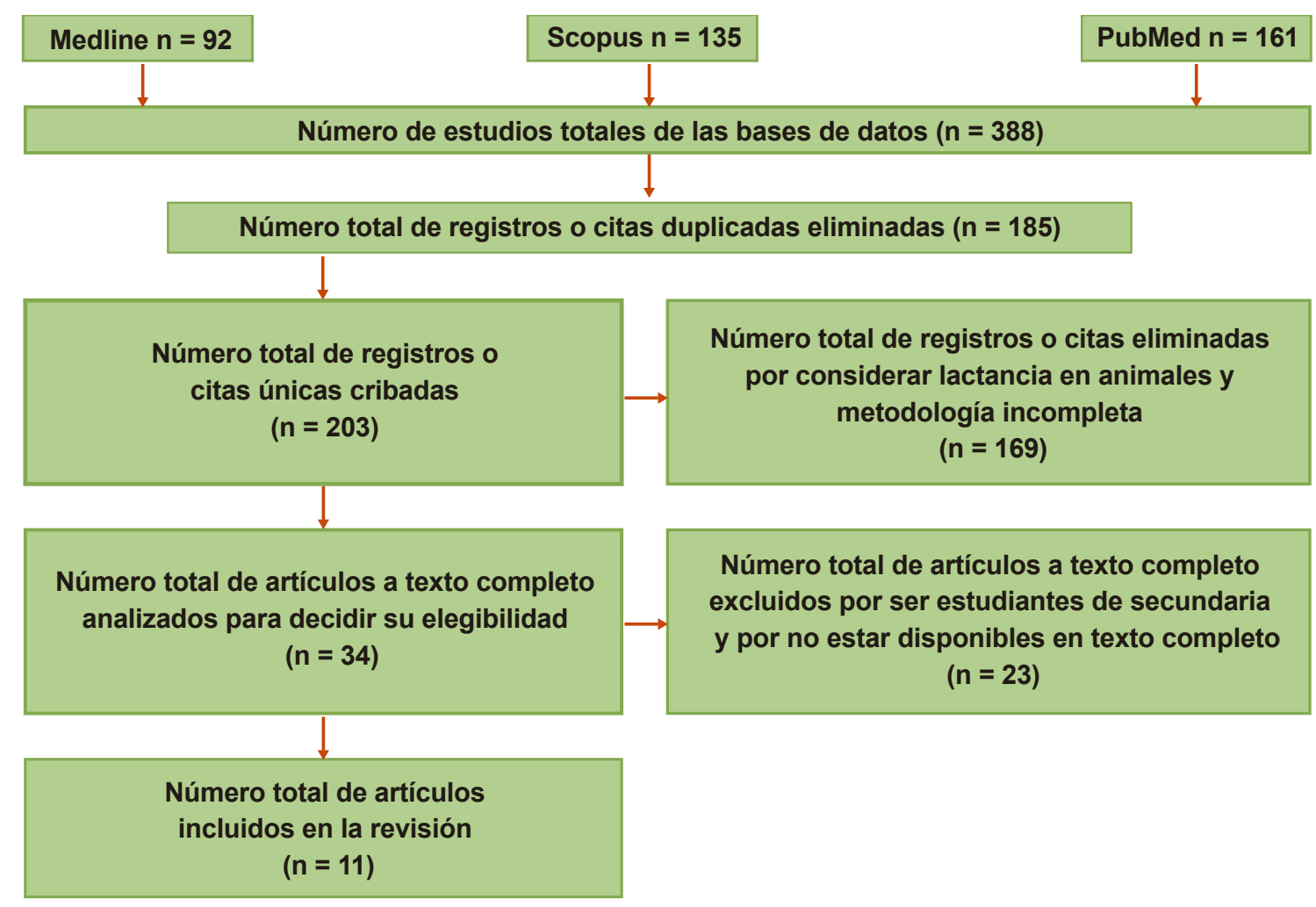

- Figura 1. Diagrama de flujo PRISMA para la selección de estudios.

Figure 1. PRISIMA flow chart for the selection of studies. 
cualitativos cuya población de estudio fueran estudiantes universitarios. Se incluyeron publicaciones de texto completo del 2014 a la fecha, en idiomas inglés y español. Se excluyeron artículos con metodología incompleta, textos que contemplan como población de estudio a las madres, así como aquellos que se referían a lactancia en animales.

Se encontraron 388 estudios cuyo título se relacionaba con los patrones de búsqueda. Se eliminaron 185 artículos repetidos en las bases de datos y publicados antes del 2014, 59 estudios se excluyeron, ya que la población blanco eran madres y no estudiantes. Se excluyeron 13 por considerar estudiantes de secundaria y no universitarios, 10 más por no estar disponibles en texto completo.

La calidad metodológica se evaluó con la escala de la base de datos de evidencia de fisioterapia (PEDro, por sus siglas en inglés: physiotherapy evidence database) (PEDro, 2012). De esta forma, se utilizaron 10 criterios: 1 ) criterios de selección especificados, 2) tamaño de muestra mayor a 500,3) instrumento validado de medición de las actitudes 0 , 4) validez y confiabilidad del estudio y análisis estadístico, 5) características de la muestra, 6) el estudio considera sólo varones, 7) actitudes frente a la LM, 8) considera beneficios en la salud, 9) año de publicación posterior a 2018 e 10) instrumento validado de medición del conocimiento. Para su evaluación a cada criterio cumplido se le asignó un "sí”, y un valor de 10 puntos. En este sentido, se evalúa en una escala del 0 al 100, donde un mayor puntaje es indicativo de mayor calidad.

Respecto a la calidad metodológica (PEdro) de los 11 artículos seleccionados, el de Heathman y col. (2019) fue el que obtuvo mayor calificación con 70 puntos, y 9 artículos más tuvieron 60 puntos (Tabla 1 ).

Respecto al tipo de estudio, 7 son de corte transversal, 2 de cohorte, 1 cualitativo y 1 que no menciona el tipo de estudio (Tabla 2). En las investigaciones incluidas en el análisis se reportó el uso de diferentes cuestionarios como el
Cuestionario sobre el comportamiento de la lactancia materna (BBQ, por sus siglas en inglés: breastfeeding behavior questionnarire), AFORM: Prueba de conocimientos sobre alimentación infantil formulario A (en inglés, infant feeding knowledge test form A), Cuestionario australiano de conocimientos y actitudes sobre la LM (ABKAQ-20 y ABKAQ-12), por sus siglas en inglés: australian breastfeeding knowlenge and attitudes questionnare), Escala de actitudes de alimentación infantil de Iowa (IIFAS, por sus siglas en inglés: iowa infant feeding attitude), y otros creados, estructurados y autoadministrados para cada estudio (Natan y col., 2018; Linares y col., 2018), en los que se consideraban preguntas con relación al conocimiento y las actitudes respecto a la LM; asimismo, encuestas estructuradas (Vandewark, 2014; Henderson y col., 2017; Heathman y col., 2019), cuestionarios estructurados (Lou y col., 2014; Mohamad y col., 2019) y entrevistas (Yang y col., 2019b) para identificar el nivel de conocimiento y/o actitudes de los estudiantes universitarios. Para la evaluación de las actitudes se utilizó con mayor frecuencia la escala IIFAS, que evalúa el nivel de actitudes respecto a la LM. La distribución de los artículos por región y años de publicación se muestra en la Figura 2, donde se destacan más artículos en Norte América y Asia; siendo el año 2019 cuando se localizaron más estudios con los criterios seleccionados. En la Tabla 3 se muestran un resumen de los artículos incluidos en la revisión.

\section{Conocimiento de los beneficios de la LM}

Beneficios para el bebé

En el estudio de Linares y col. (2018), casi la totalidad de los participantes estuvieron de acuerdo en que la LM es beneficiosa para el bebé y que su práctica reduce el riesgo de enfermedad (99 \%). Mientras que en la investigación de Heathman y col. (2019) refirieron que más del $50 \%$ de los participantes no identificaron los beneficios para la salud del infante, incluido un menor riesgo de sobrepeso y obesidad, infecciones de oído y alergias alimentarias. Un $72 \%$ no logró distinguir la relación entre la leche materna y la disminución del riesgo de aparición de diarrea. Esta 
diferencia en el nivel de conocimiento sobre dos (Linares y col., 2018). Por otra parte, el $84 \%$ los beneficios de la LM para el binomio se debe a la formación especializada recibida por los de los estudiantes encuestados por Lou y col. estudiantes de enfermería del estudio llevado a (2014) en Asia, reconocen que la LM ayuda a prevenir infecciones respiratorias en los bebés cabo en el estado de Kentucky, en Estados Uni- y continúan los beneficios después del destete.

Tabla 1. Resultados del análisis de calidad de los artículos seleccionados sobre el conocimiento y actitudes respecto a los beneficios de la lactancia materna en la salud del binomio madre-hijo en estudiantes universitarios.

Table 1. Results of the quality analysis of the selected articles on the knowledge and attitudes regarding the health benefits of breastfeeding in the mother-child binomial in university students.

\begin{tabular}{|c|c|c|c|c|c|c|c|c|c|c|c|}
\hline $\begin{array}{l}\text { Autor, } \\
\text { año de } \\
\text { publicación }\end{array}$ & 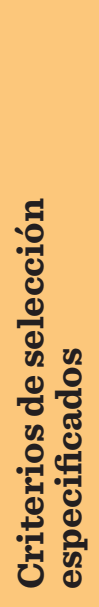 & 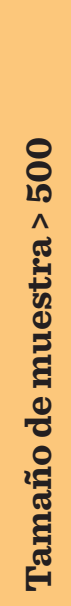 & 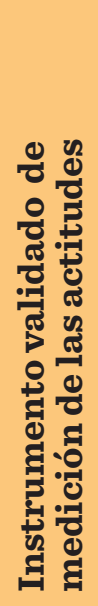 & 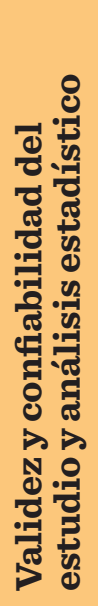 & 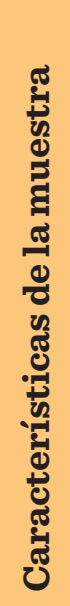 & 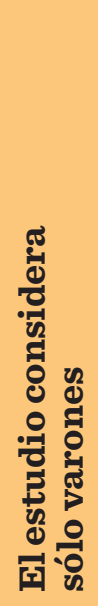 & 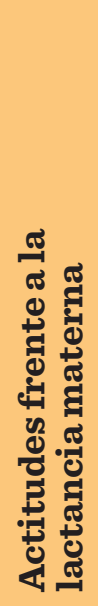 & 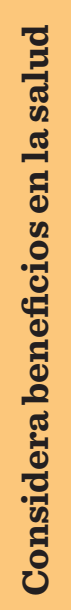 & 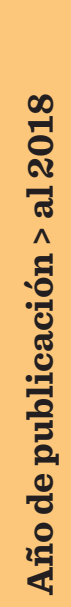 & 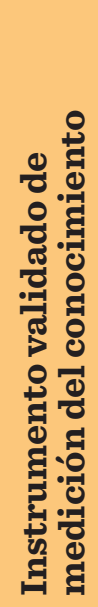 & సేత్ \\
\hline $\begin{array}{l}\text { Hamade y col. } \\
\text { (2014) }\end{array}$ & Sí & & Sí & Sí & Sí & & Sí & & & Sí & 60 \\
\hline $\begin{array}{l}\text { Lou y col. } \\
\text { (2014) }\end{array}$ & Sí & & & Sí & Sí & & Sí & & & & 40 \\
\hline $\begin{array}{l}\text { Vandewark } \\
(2014)\end{array}$ & Sí & & Sí & Sí & Sí & & Sí & Sí & & & 60 \\
\hline $\begin{array}{l}\text { Jefferson } \\
\text { (2017) }\end{array}$ & Sí & Sí & Sí & Sí & Sí & & Sí & & & & 60 \\
\hline $\begin{array}{l}\text { Henderson y col. } \\
\text { (2017) }\end{array}$ & Sí & Sí & Sí & Sí & Sí & & Sí & & & & 60 \\
\hline $\begin{array}{l}\text { Yang y col. } \\
\text { (2019b) }\end{array}$ & Sí & & & Sí & Sí & & Sí & Sí & Sí & & 60 \\
\hline $\begin{array}{l}\text { Linares y col. } \\
\text { (2018) }\end{array}$ & Sí & Sí & & Sí & Sí & & & Sí & Sí & & 60 \\
\hline $\begin{array}{l}\text { Natan y col. } \\
\text { (2018) }\end{array}$ & Sí & & & Sí & Sí & & Sí & Sí & Sí & & 60 \\
\hline $\begin{array}{l}\text { Yang y col. } \\
\text { (2019a) }\end{array}$ & Sí & & Sí & Sí & Sí & & & & Sí & Sí & 60 \\
\hline $\begin{array}{l}\text { Heathman y col. } \\
\text { (2019) }\end{array}$ & Sí & Sí & & Sí & Sí & Sí & Sí & & Sí & & 70 \\
\hline $\begin{array}{l}\text { Mohamad y col. } \\
\text { (2019) }\end{array}$ & Sí & & & Sí & Sí & & Sí & Sí & Sí & & 60 \\
\hline
\end{tabular}




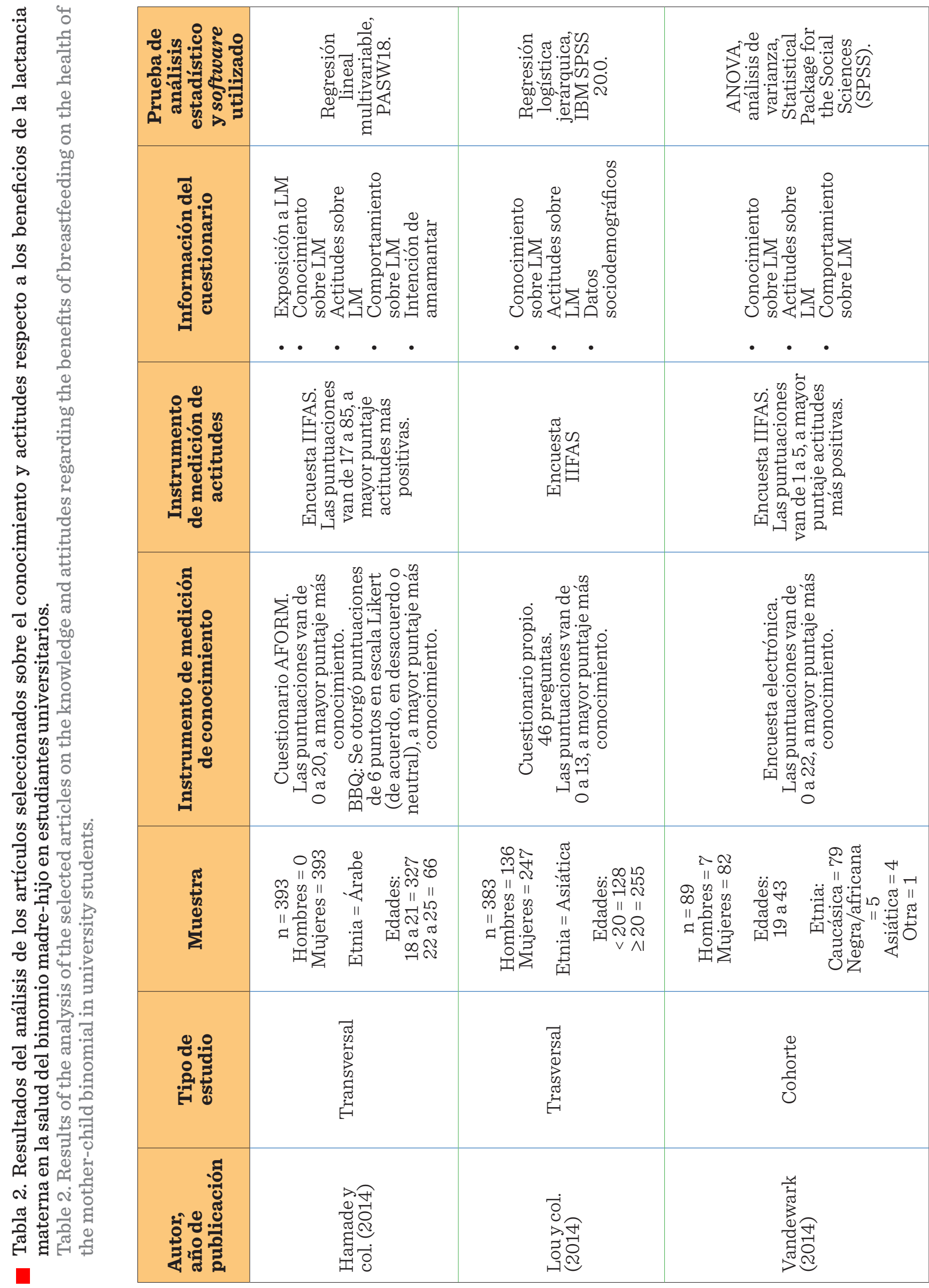




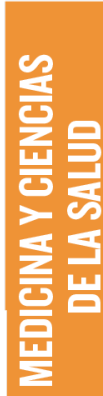

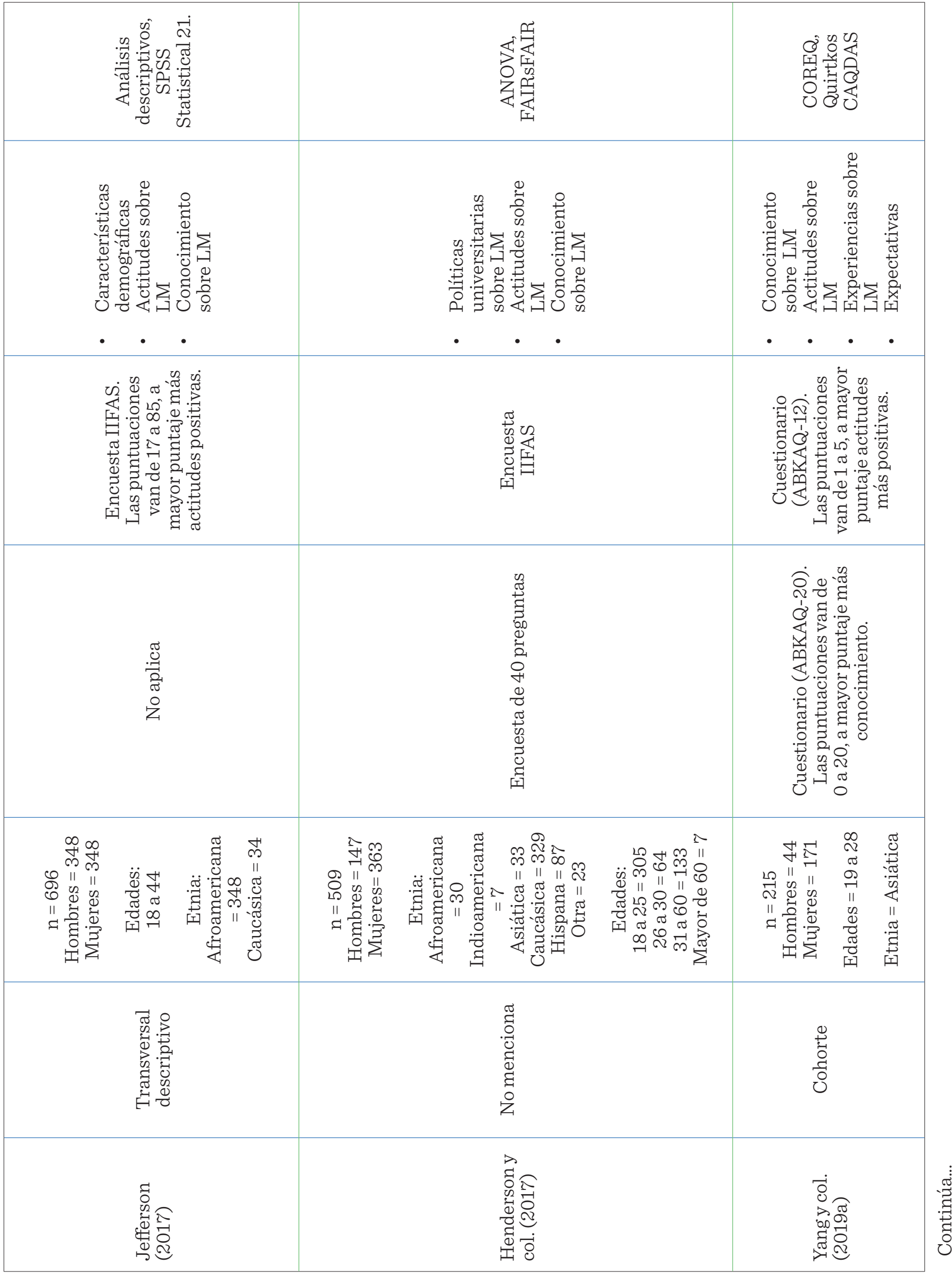




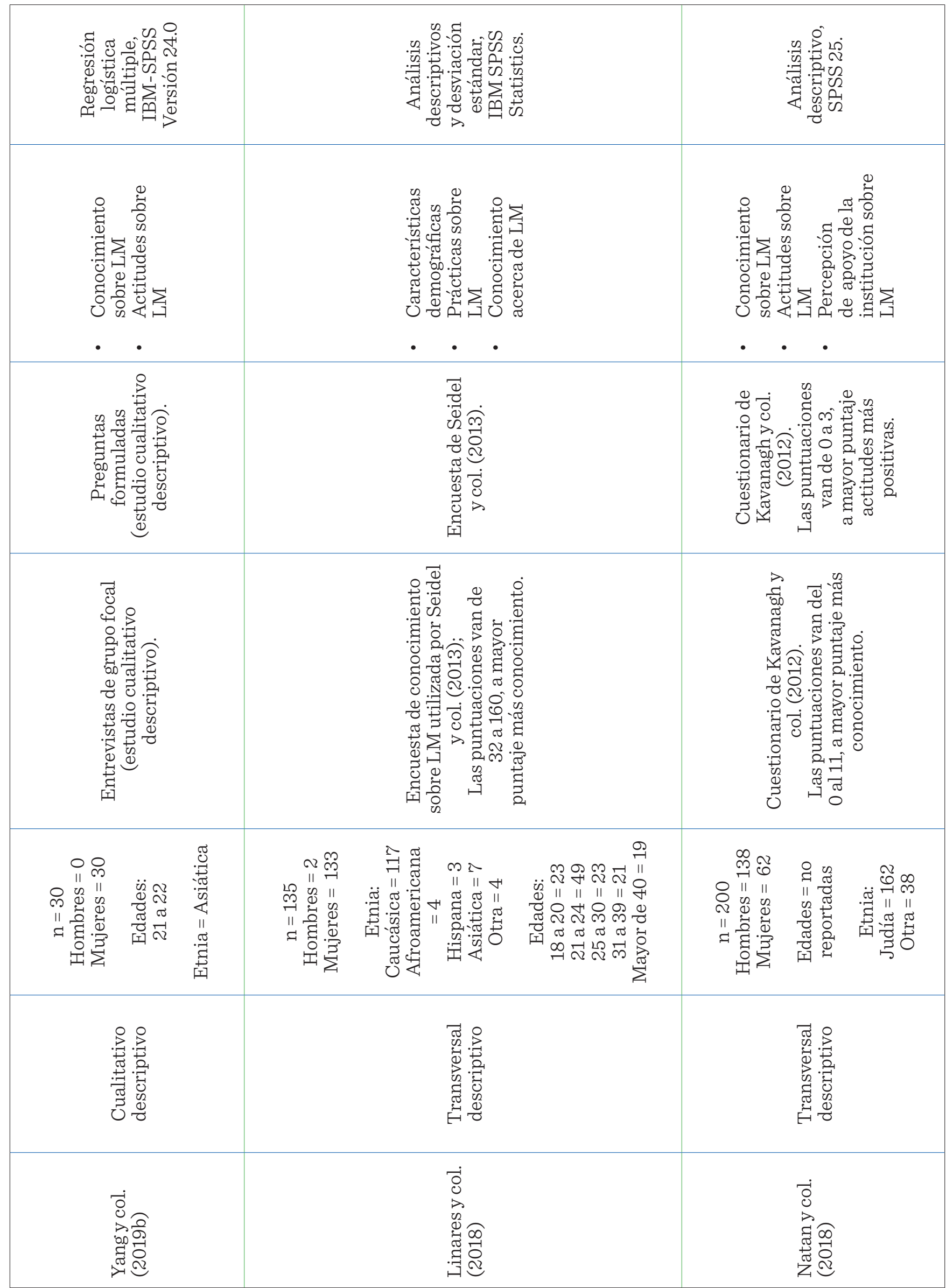




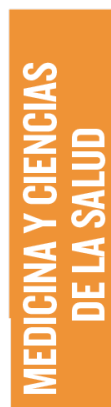

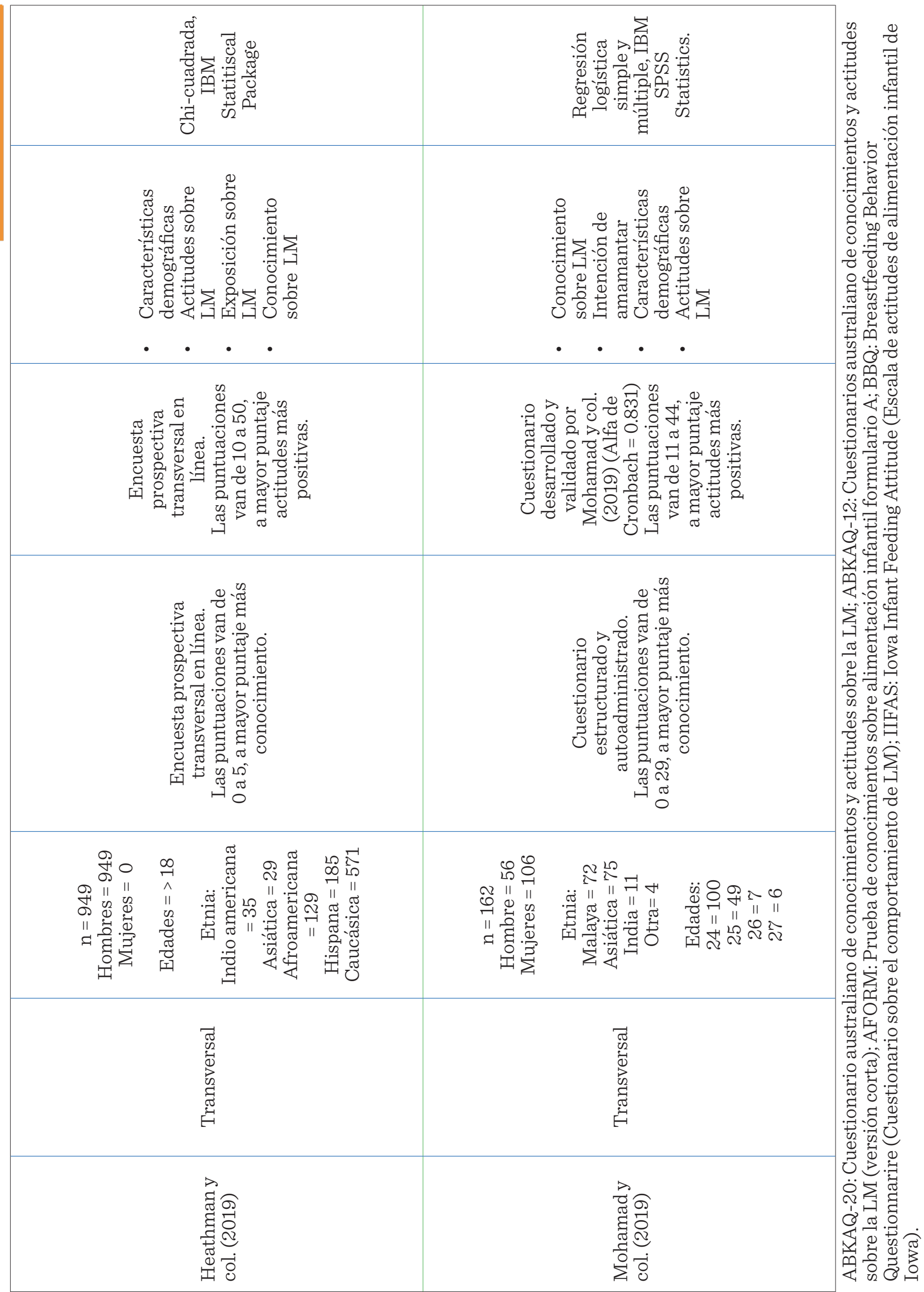




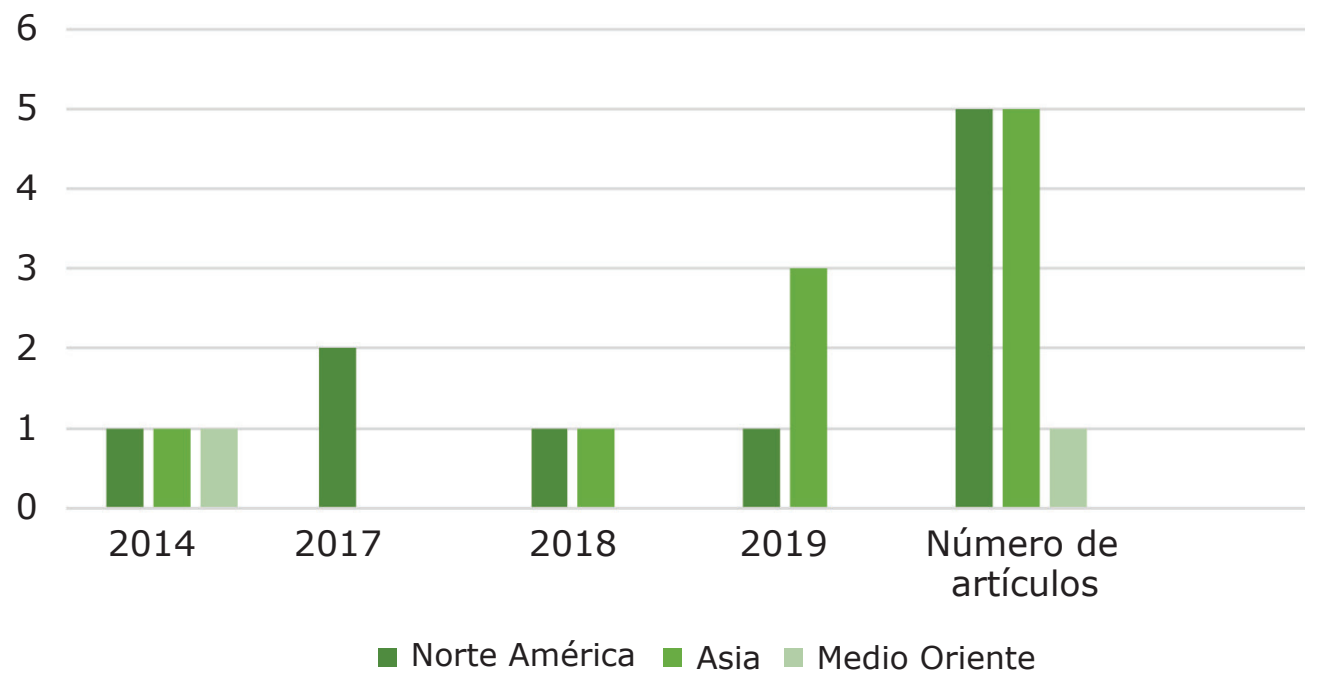

- Figura 2. Distribución de los artículos por región y año de publicación.

Figure 2. Distribution by region and year of publication.

Mohamad y col. (2019) reportaron un $95 \%$ de aciertos en el reconocimiento en la reducción del riesgo de infección pulmonar, protección contra las alergias, buen desarrollo de los dientes y la encía del bebé, así como aumento de la inteligencia.

\section{Beneficios en la madre}

Universitarios asiáticos identificaron que las mujeres que han amamantado tienen un menor riesgo de padecer cáncer de mama (Yang y col., 2019a). Estos resultados son similares a los encontrados por Mohamad y col. (2019). Por su parte, Hamade y col. (2014) reportaron que alrededor de un $65 \%$ de los participantes en Siria y Líbano, no eran conscientes de la disminución del riesgo de hemorragia posparto con la LM; así también, registran que la LM exclusiva ayuda a estimular la contracción uterina en la madre y prevenir la congestión mamaria. Finalmente, estudiantes de Norteamérica (Linares y col., 2018), consideraron que la LM beneficia la salud de la madre reduciendo el riesgo de enfermedades y ayuda a la pérdida de peso materna.

\section{Edad}

Lou y col. (2014), identificaron que estudiantes jóvenes (< 20 años) tenían mayor conocimiento sobre la duración de la LM, los beneficios para la salud del bebé y la madre, también sobre los vínculos afectivos y las técnicas adecuadas para llevar a cabo la LM. Sin embargo, Heathman y col. (2019) documentaron la edad de los estudiantes como un predictor de la puntuación de conocimiento sobre la LM, encontrando que a mayor edad, existía más conocimiento. En este mismo sentido, Henderson y col. (2017) reportaron que los encuestados más jóvenes obtuvieron puntuaciones más bajas en el nivel de conocimiento que sus contrapartes mayores. Dicha situación puede tener relación con lo descrito por Linares y col. (2018) y Natan y col. (2018), quienes identificaron más conocimiento en población mayor, sobre todo cuando los estudiantes ya habían sido padres de familia, detectaron diferencias significativas entre este sector y los que aún no tenían hijos.

\section{Sexo}

Respecto al nivel de conocimiento por sexo hay reportes diversos, por un lado, en el estudio de Lou y col. (2014), los hombres obtuvieron puntuaciones más altas que las mujeres, a diferencia del estudio presentado por Heathman 


\begin{tabular}{|c|c|c|}
\hline 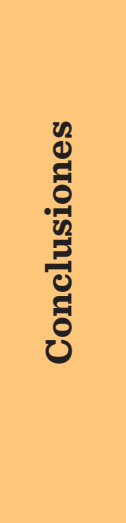 & 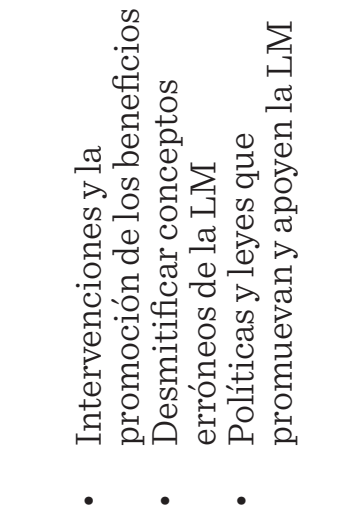 & 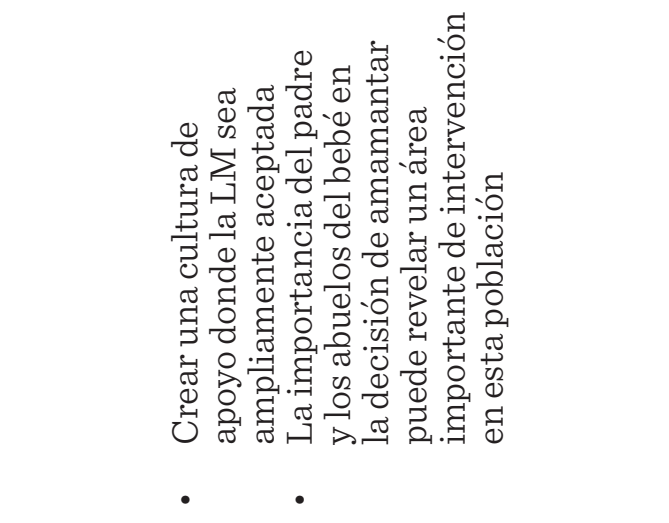 \\
\hline 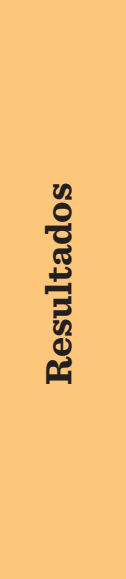 & 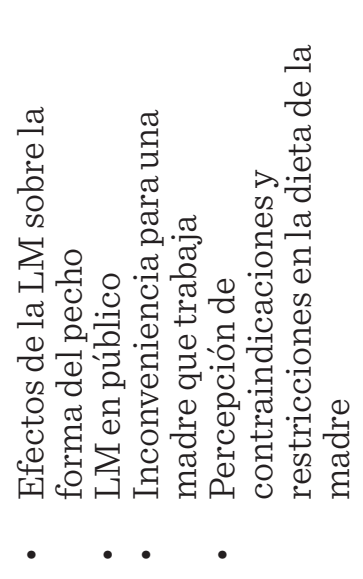 & 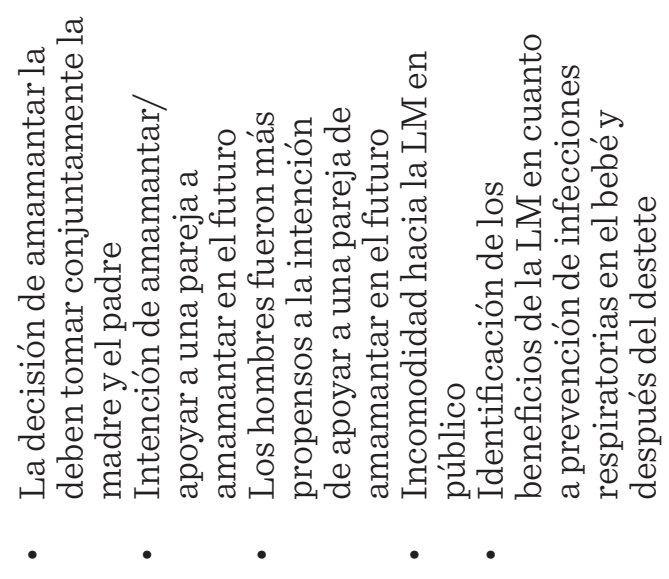 \\
\hline 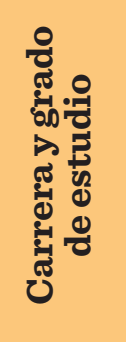 & 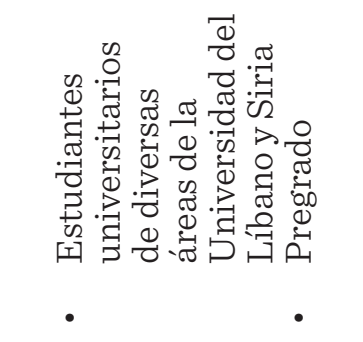 & 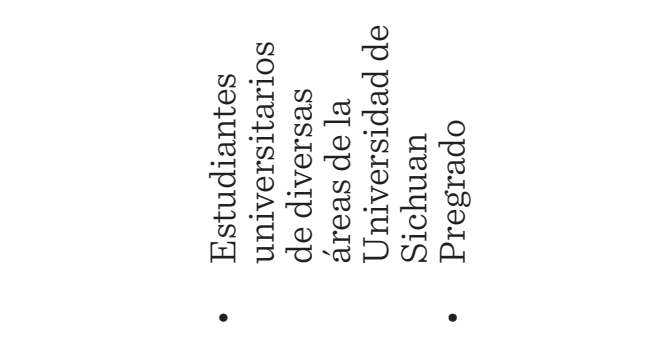 \\
\hline 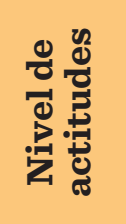 & 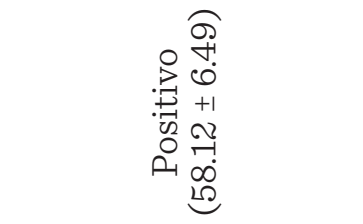 & 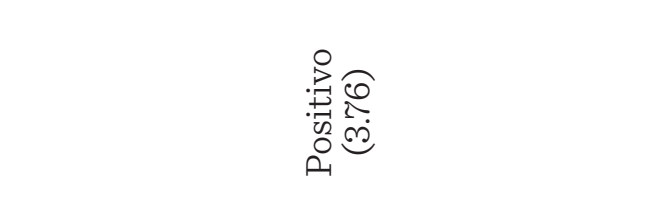 \\
\hline 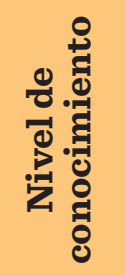 & 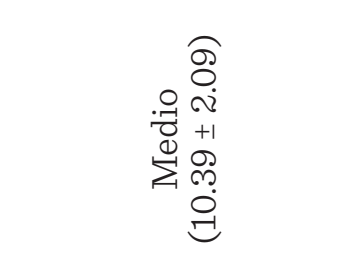 & 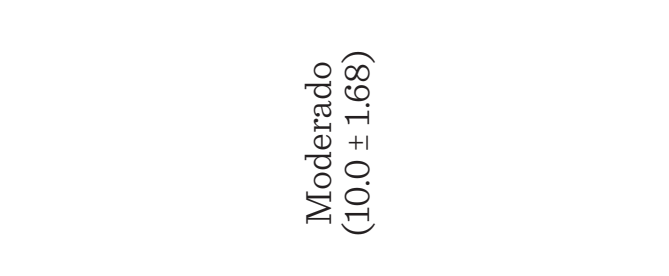 \\
\hline 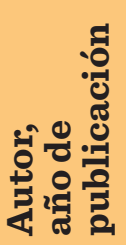 & 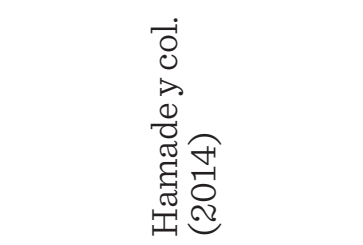 & 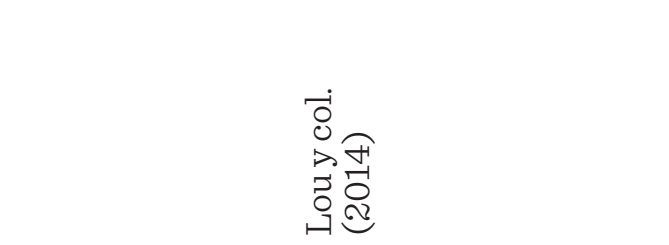 \\
\hline
\end{tabular}




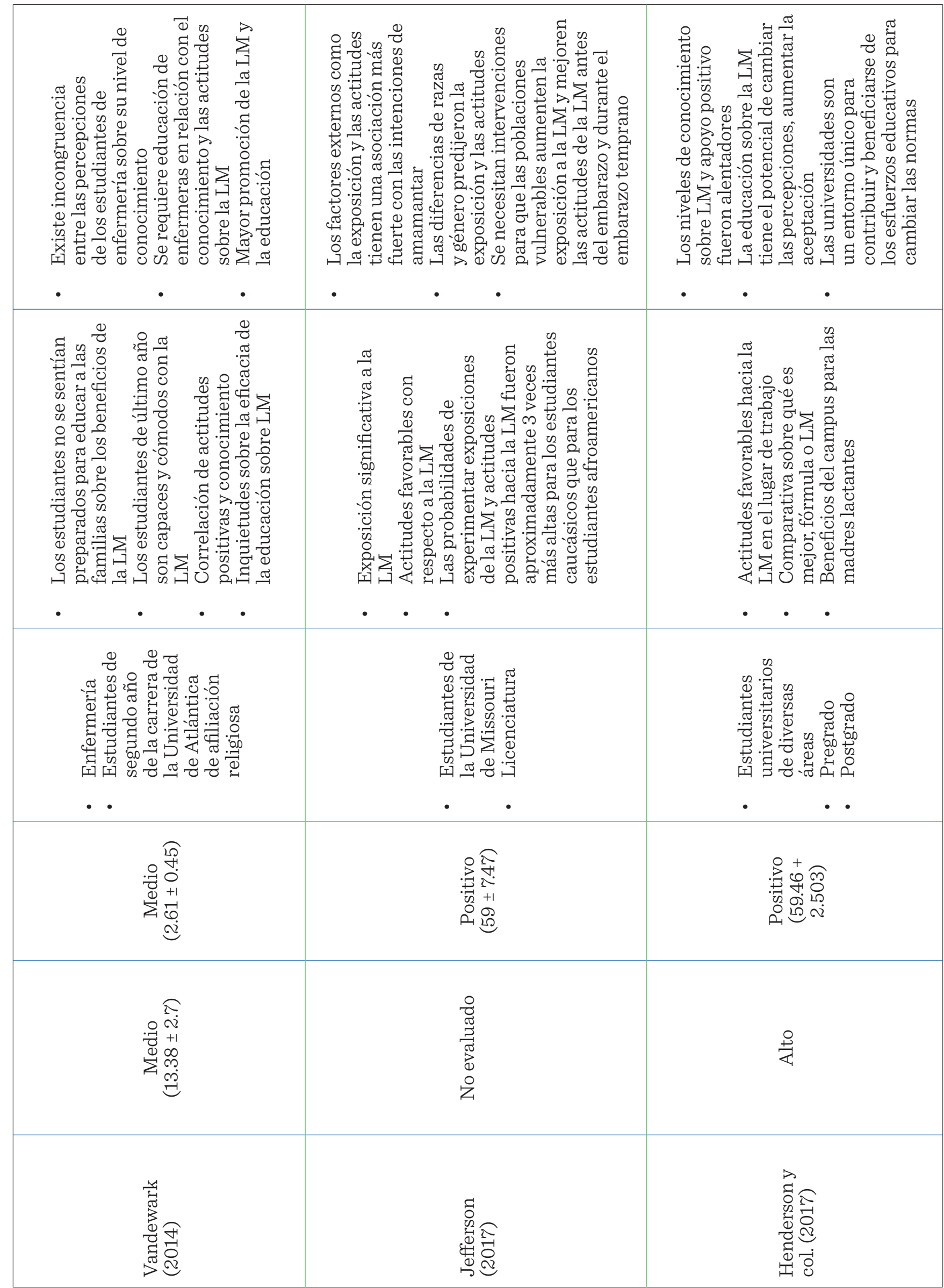




\begin{tabular}{|c|c|c|}
\hline 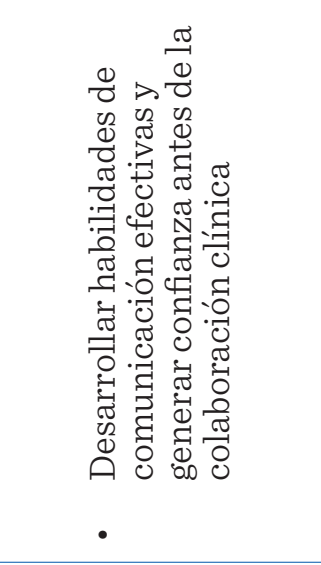 & 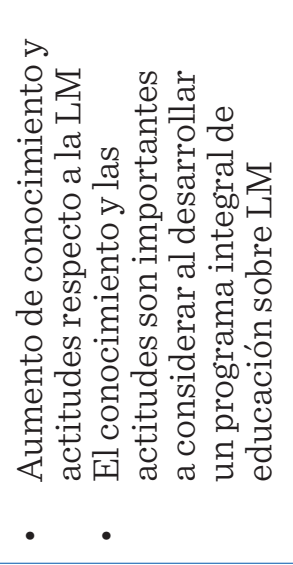 & 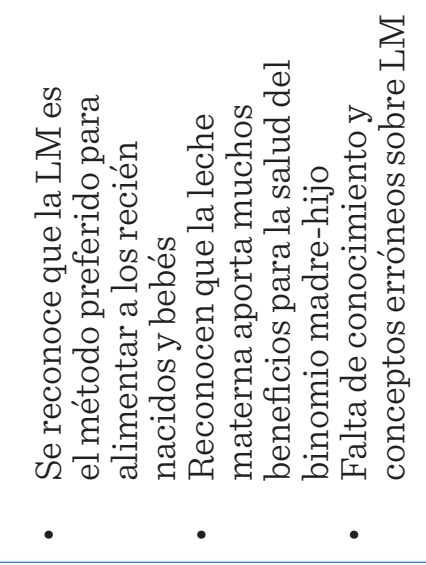 \\
\hline 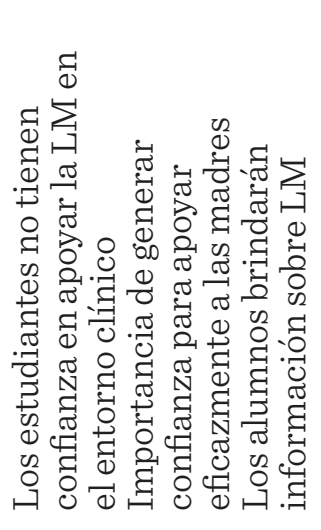 & 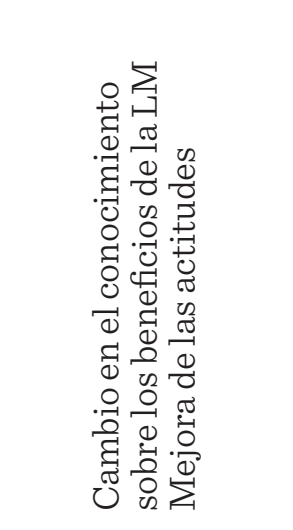 & 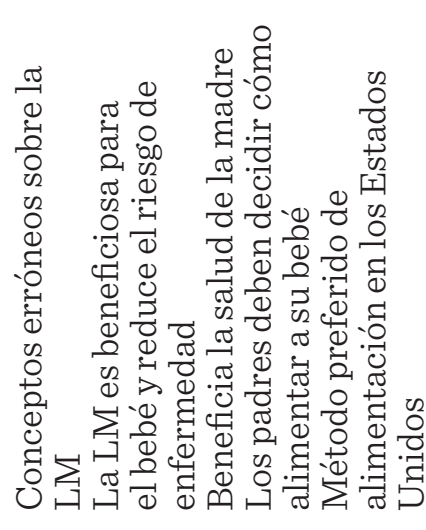 \\
\hline . $\quad \cdot \quad \cdot$ & • $\quad \bullet$ & $\begin{array}{llll}\bullet & \bullet & \bullet & \bullet\end{array}$ \\
\hline 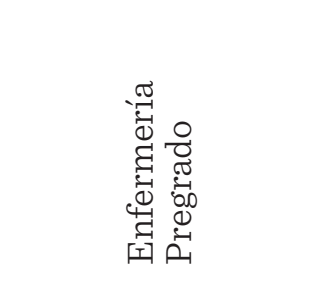 & 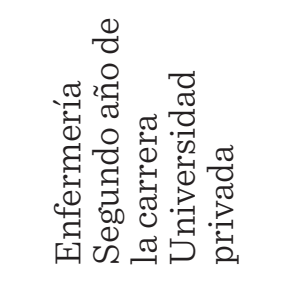 & 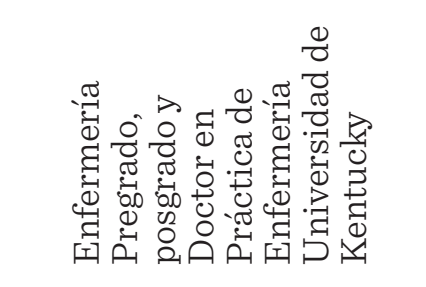 \\
\hline$\cdot \cdot$ & • • • & • $\quad$ ・ \\
\hline 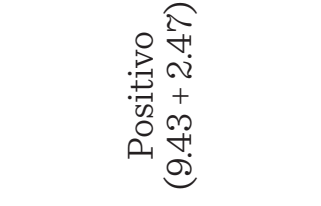 & 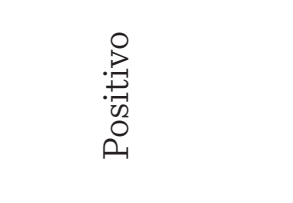 & $\begin{array}{l}\text { i⿱ } \\
\text { i } \\
\sum_{i}^{\infty}\end{array}$ \\
\hline 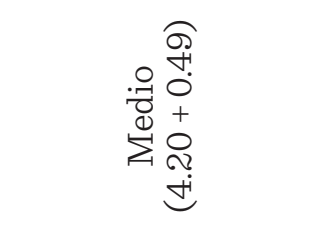 & 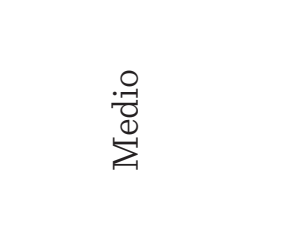 & . \\
\hline 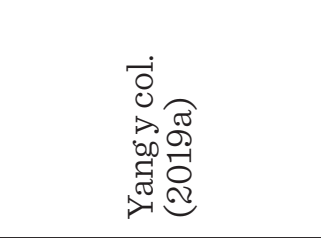 & 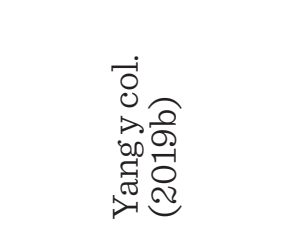 & 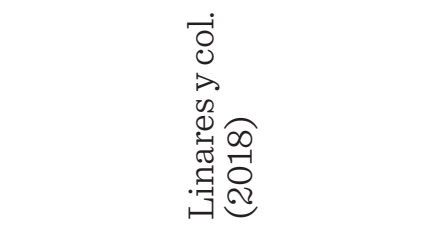 \\
\hline
\end{tabular}




\begin{tabular}{|c|c|c|}
\hline 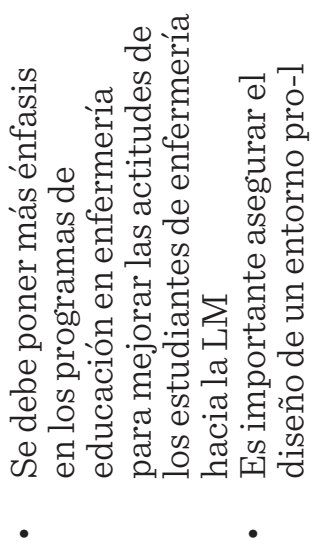 & 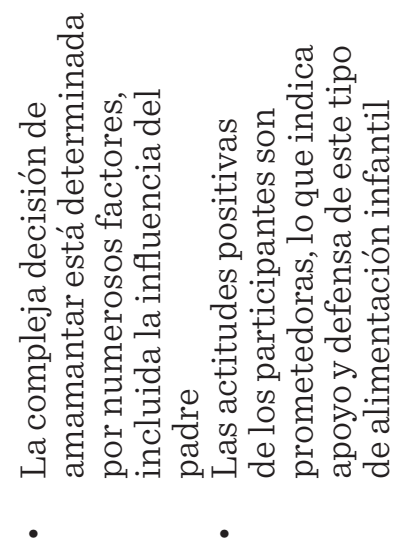 & 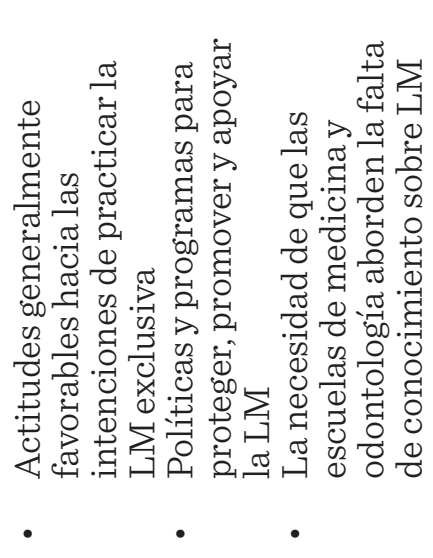 \\
\hline 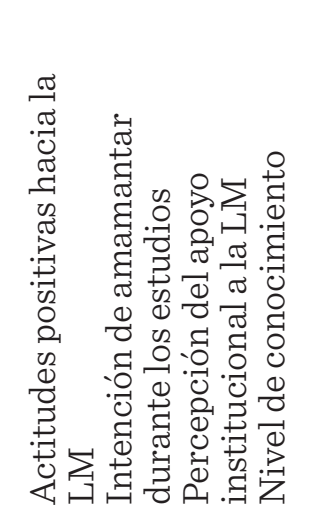 & 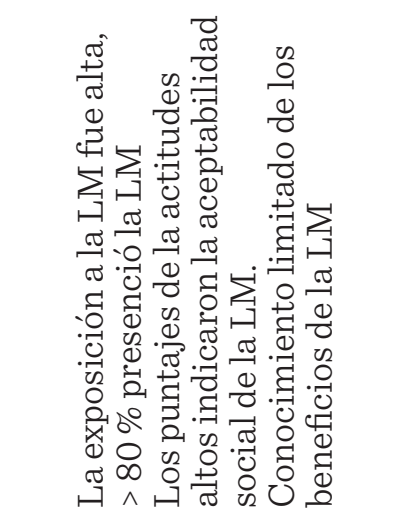 & 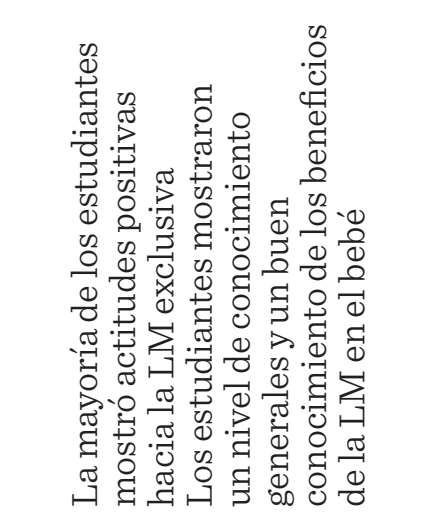 \\
\hline $\begin{array}{llll}\cdot & \cdot & \cdot & \cdot\end{array}$ & • $・$ ・ & - \\
\hline 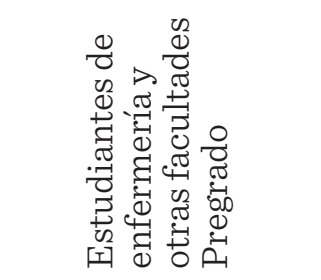 & 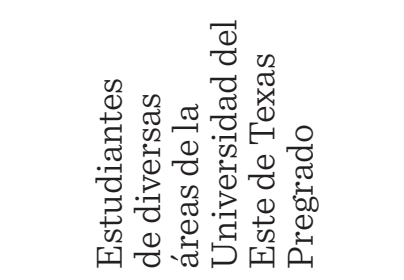 & 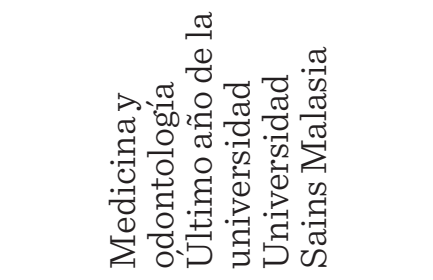 \\
\hline - $\quad$. & - $\quad \bullet$ & • $\quad \cdot \quad \cdot$ \\
\hline 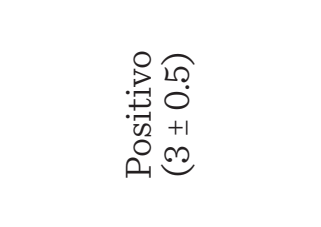 & 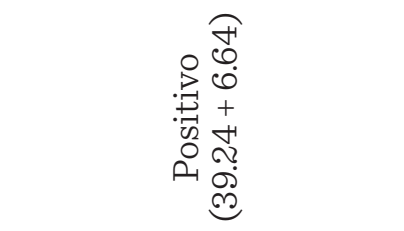 & 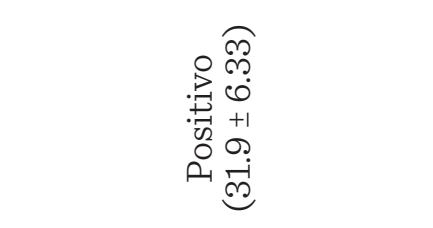 \\
\hline 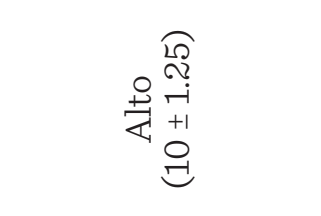 & 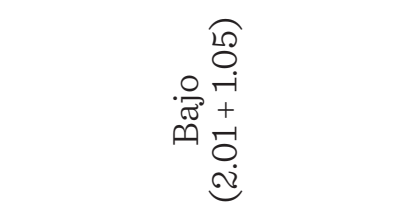 & 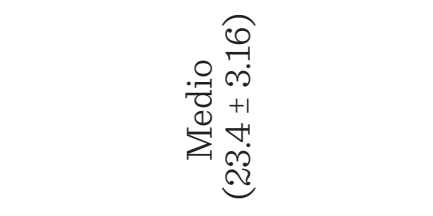 \\
\hline 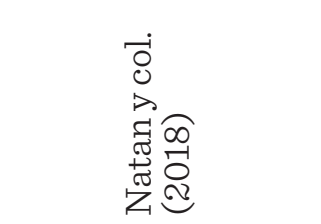 & 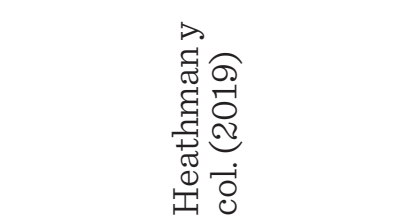 & 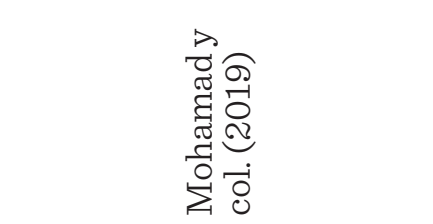 \\
\hline
\end{tabular}


y col. (2019) donde solo participaron hombres y registraron puntuaciones bajas. Por su parte Henderson y col. (2017) no reportaron diferencias significativas en la comparación entre hombres y mujeres.

\section{País o etnia}

Con respecto a la pertenencia étnica, los estudiantes caucásicos (65\%) que participaron en el estudio de Henderson y col. (2017) tenían un nivel de conocimiento alto y fueron capaces de identificar los beneficios de la LM en la salud del binomio madre e hijo, el incremento del costo de la alimentación con fórmula y reconocieron a la leche materna como el alimento ideal para los bebés. Heathman y col. (2019), por su parte identificaron que la etnia no era indicador del conocimiento sobre la LM, ya que los participantes caucásicos, afroamericanos y asiáticos tenían un nivel de conocimiento bajo. En otros estudios cuya muestra de población es perteneciente a la etnia asiática se mostró un nivel de conocimiento medio sobre la LM (Lou y col., 2014; Mohamad y col., 2019; Yang y col., 2019a; 2019b).

En cuanto al conocimiento general, se encontró que el $70 \%$ de los estudiantes de Malasia (Mohamad y col., 2019) estuvieron de acuerdo en que la LM es menos costosa que la alimentación con fórmula y un $60.5 \%$ mencionó que la LM puede ahorrar gastos familiares. Por otro lado, más del $80 \%$ de los estudiantes participantes de Norteamérica y Asia reconocieron a la LM como el alimento más saludable para el bebé (Lou y col., 2014 y Jefferson, 2017). La mayoría (70 \%) de los estudiantes estadounidenses de enfermería consideraban la LM como el método de alimentación preferido para un bebé e informaron que la LM es normal para sus familias (Linares y col., 2018). Por otro lado, Lou y col. (2014) en su investigación mostraron que el $91.8 \%$ de los estudiantes asiáticos refieren que la LM ayudará a la madre a sentirse más cerca de su bebé.

Sobre el conocimiento de la duración de la LME durante los primeros 6 meses de vida, únicamente el $16.12 \%$ de universitarios nor- teamericanos conocían el periodo recomendado (Heathman y col., 2019), a diferencia del estudio de Mohamad y col. (2019), en donde la mayoría de los estudiantes de Malasia sabían que, la LM debe iniciarse dentro de los $30 \mathrm{~min}$ posteriores al parto y que la duración correcta de la LME es de 6 meses.

\section{Área de estudio}

Acerca de las áreas de estudio, existió diferencia en el nivel de conocimiento que tenían los estudiantes del área de la salud sobre la LM con relación a los estudiantes de otras áreas no médicas. Yang y col. (2019a) expresaron que el nivel de conocimiento relacionado con la LM puede aumentar por la instrucción teórica (aula y laboratorio) que reciben los estudiantes profesionales de la salud. En ese sentido, Natan y col. (2018), encontraron que los estudiantes de enfermería tenían un nivel de conocimiento más alto que los estudiantes de áreas diferentes a la salud, sin embargo, es necesario tomar en consideración que Yang y col. (2019b) sugieren que el aprendizaje en el aula no prepara adecuadamente a los estudiantes para apoyar a las madres con la LM, por ende, estudiantes de enfermería informaron que para mejorar el nivel de conocimiento sobre la LM necesitan tener más ejercicios prácticos antes de iniciar el ejercicio clínico. Los participantes del estudio de Heathman y col. (2019) no pertenecientes al área de la salud obtuvieron un nivel de conocimiento bajo, reconocieron la asequibilidad de la LM, pero no identificaron que la nutrición ofrecida por la leche materna es superior a la alimentación con leche de fórmula, además de los múltiples beneficios para la salud de la madre y el hijo.

\section{Actitudes de los beneficios de la LM}

Respecto a las actitudes de los beneficios de la LM en la vida social y laboral de las madres, Heathman y col. (2019) refirieron que la mayoría de los encuestados (82 \%) manifestaron estar en desacuerdo con que la LM atará a la madre e interferirá en su vida social. Por su parte, en el estudio de Henderson y col. (2017) aproximadamente una cuarta parte (22.8 \%) de los participantes consideraban que la for- 
mula era mejor que la lactancia materna cuando una madre regresaba al trabajo.

Yang y col. (2019b) documentaron las actitudes respecto al consumo de leche materna, se reportó que el $75.8 \%$ de su población de estudio se inclinó por la igualdad de la fórmula y la leche materna como alimentos saludables. Sin embargo, las mujeres (54\%) del estudio de Jefferson (2017), estuvieron en desacuerdo con la afirmación de que la fórmula es tan saludable como la leche materna, en comparación con el $42 \%$ de los hombres.

\section{Edad}

En lo que respecta al nivel de actitudes sobre la LM, Mohamad y col. (2019), indicaron que la mayoría de los adultos jóvenes (93.2 \%) tenían actitudes positivas. En contraste con los resultados de Henderson y col. (2017) en donde los encuestados de mayor edad mostraron actitudes de comprensión y obtuvieron un nivel más alto que los estudiantes más jóvenes.

Sexo

Con relación al nivel de actitudes sobre la LM por sexo, Lou y col. (2014) mostraron diferencias según el sexo, ya que los hombres obtuvieron un nivel más alto que las mujeres. Lo mismo documentaron Heathman y col. (2019), en donde más del $80 \%$ de los varones participantes tuvieron un nivel positivo de actitudes hacia la LM.

Por otro lado, Jefferson (2017), reportó que las mujeres tenían actitudes más positiva en comparación con los hombres, sin embargo, esta diferencia no fue estadísticamente significativa. Cabe resaltar que culturalmente la mujer sigue teniendo un rol primordial en ejercicio de la LM en comparación con los hombres (Martínez y col., 2017), aunque esto no implica que el hombre no tenga un rol importante a nivel social, por lo cual es necesario que tanto hombres como mujeres conozcan los beneficios de la LM para el mejoramiento de la salud de generaciones futuras.

En relación con las actitudes, el uso de la escala IIFAS en varios estudios permitió identificar diferencias en sus niveles. Hamade y col. (2014) reportaron que las actitudes positiva se mantenía por encima de la media (58.12 $\pm 6.49 \%)$ tanto para hombres como para mujeres y no encontró diferencias significativas por sexo. Por otro lado, Henderson y col. (2017) encontraron diferencias significativas respecto al sexo, teniendo menor puntuación los hombres (59.86 \%) en comparación con las mujeres (61.86 \%). Caso similar fue documentado por Jefferson (2017), donde también las mujeres obtuvieron una puntuación más alta con respecto a las actitudes de amamantar (60\%).

\section{País o etnia}

Referente a las actitudes con base en la pertenencia étnica, Hamade y col. (2014) mencionaron que los estudiantes árabes (libaneses y sirios) consideraban la LM como uno de los grandes placeres de la maternidad, registrado como un nivel positivo en las actitudes sobre la LM. Estudiantes asiáticos mantuvieron actitudes positivas en los beneficios de la LM, el predominio de la LM sobre el uso de fórmula y biberón, asimismo consideraron que la LME es la mejor opción para las madres que trabajan (Lou y col., 2014; Mohamad y col., 2019; Yang y col., 2019a; 2019b).

La mayoría de los estudiantes de Malasia (63 \%) incluidos en el estudio de Mohamad y col. (2019) manifestaron estar de acuerdo en que LME puede ser difícil en lugares públicos. Por otro lado, Lou y col. (2014) identificaron que el $58.2 \%$ de los estudiantes chinos sentían vergüenza de que sus parejas amamantaran en público, así mismo la aceptabilidad de la LM en público fue baja (34.7 \%), y Jefferson (2017) reportó que los estudiantes caucásicos tenían unas actitudes positiva sobre la LM, contrario a los estudiantes afroamericanos que mostraron niveles 3 veces menores, obteniendo como resultado unas actitudes media. Por su parte, Linares y col. (2018), señalaron en su investigación que los estudiantes caucásicos (85.9 \%) presentaron un nivel de actitudes medio, pues consideraban que la alimentación con biberón es más fácil y no reconocen que la leche de fórmula conduce a la obesidad. 
Los niveles de conocimiento y actitudes por región se muestran en la Figura 3. Se encontró que solo un artículo (Jefferson, 2017) no estaba relacionado con el nivel de conocimiento.

\section{Área de estudio}

Según Natan y col. (2018) no se encontró diferencia entre los estudiantes del área de la salud y los de otras áreas, ambos grupos coincidían en la evaluación positiva de las actitudes hacia la LM. Esto concuerda con lo reportado por Vandewark (2014) donde se observó que los estudiantes del área de la salud presentaron unas actitudes positivas. Al igual que Heathman y col. (2019), registraron actitudes positivas hacia la LM en estudiantes no pertenecientes al área de la salud. Por su parte, Yang y col. (2019a) encontraron que el nivel de las actitudes sobre la LM en universitarios del área de la salud aumentó con la educación teórica (5\%) y la práctica clínica (5\%).

En el estudio presentado por Yang y col. (2019a) se describe que los planes de estudio del área de la salud, abordan el conocimiento y acti- tudes de la LM en la asignatura de salud materno-infantil, a través de clases teóricas, aprendizaje simulado y práctica de habilidades en el laboratorio, busca que los estudiantes adquieran conocimiento y conceptos básicos sobre la LM, incluyendo los beneficios en la salud de la madre e hijo, posturas y técnicas para amamantar, duración de la LME, vínculos afectivos, así como las barreras sociales y problemas comunes de la LM. Es primordial que los profesionales y estudiantes de las áreas de la salud tengan unas actitudes positiva hacia la LM y el conocimiento y habilidades suficientes para orientar y promover la práctica de la LM (Vandewark, 2014; Linares y col., 2018; Natan y col., 2018; Yang y col., 2019b).

Los hallazgos por edad, sexo y área de estudio se muestran en la Figura 4, observándose en general un nivel de conocimiento medio (4a), pero un nivel de actitudes positivo hacia la lactancia (4b).

\section{Barreras de la lactancia materna}

Jefferson (2017) manifiestó que más estudian-

Nivel de conocimiento y actitudes por región

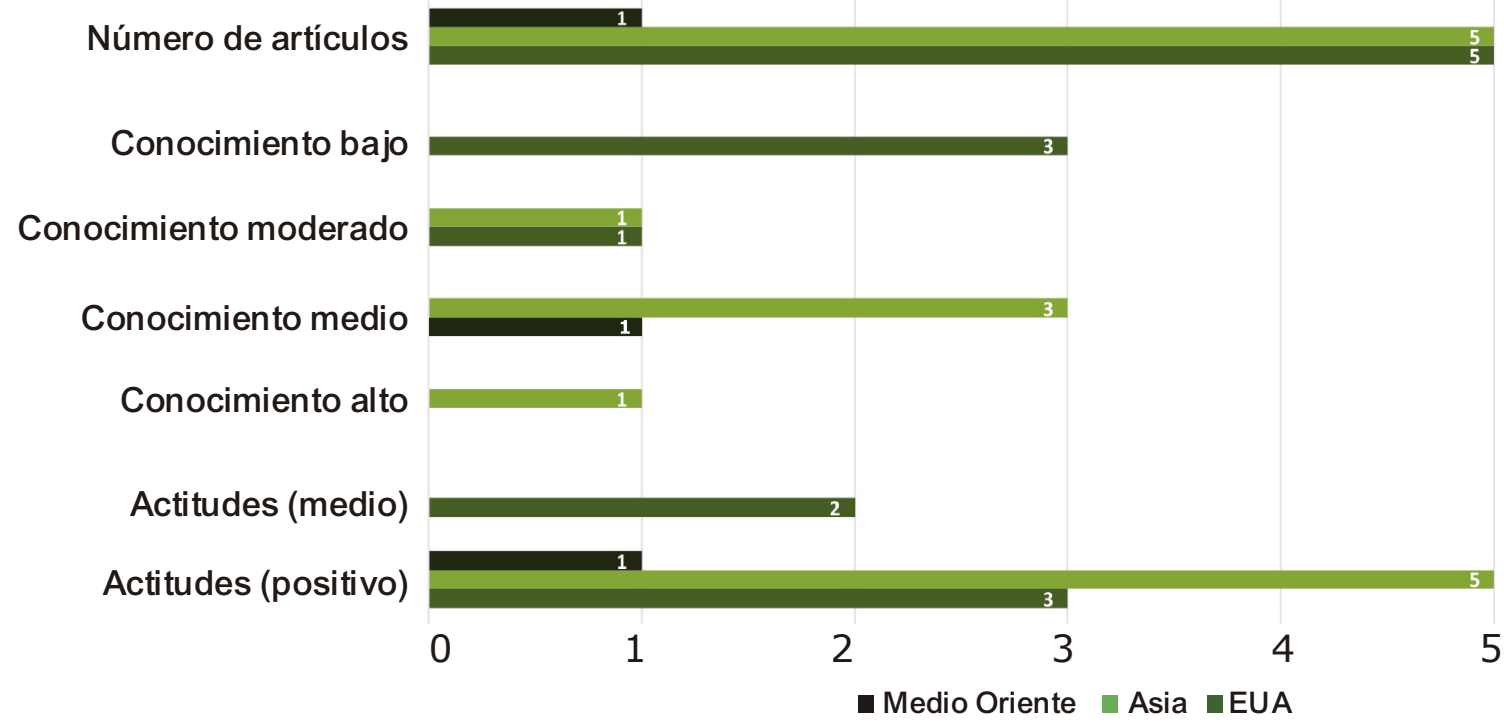

\section{5}

- Figura 3. Número de reportes de los niveles de conocimiento y actitudes por región. Figure 3. Number of reports of the levels of knowledge and attitude by region. 
a) Nivel de conocimiento por edad, sexo y área de estudio

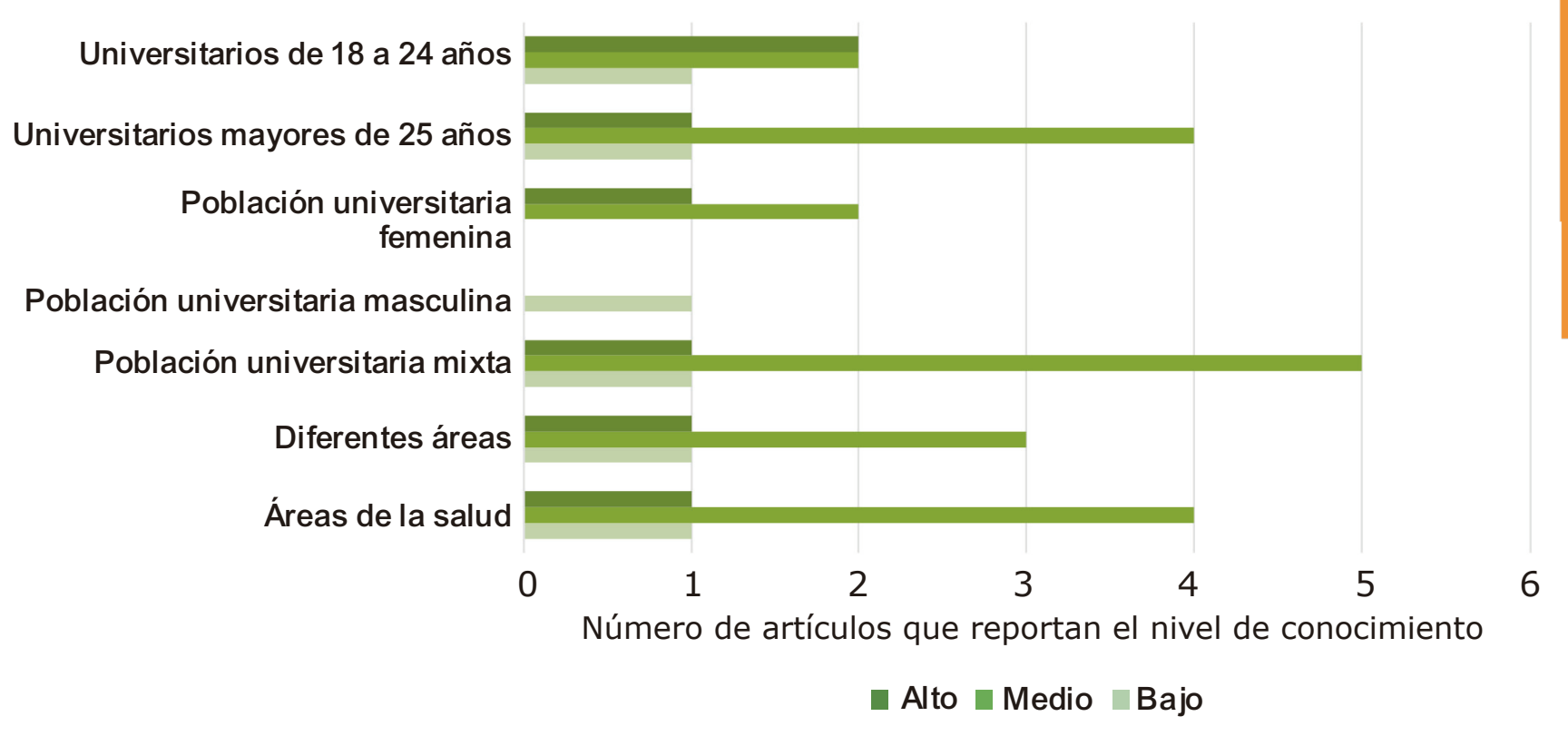

b) Nivel de actitudes por edad, sexo y área de estudio

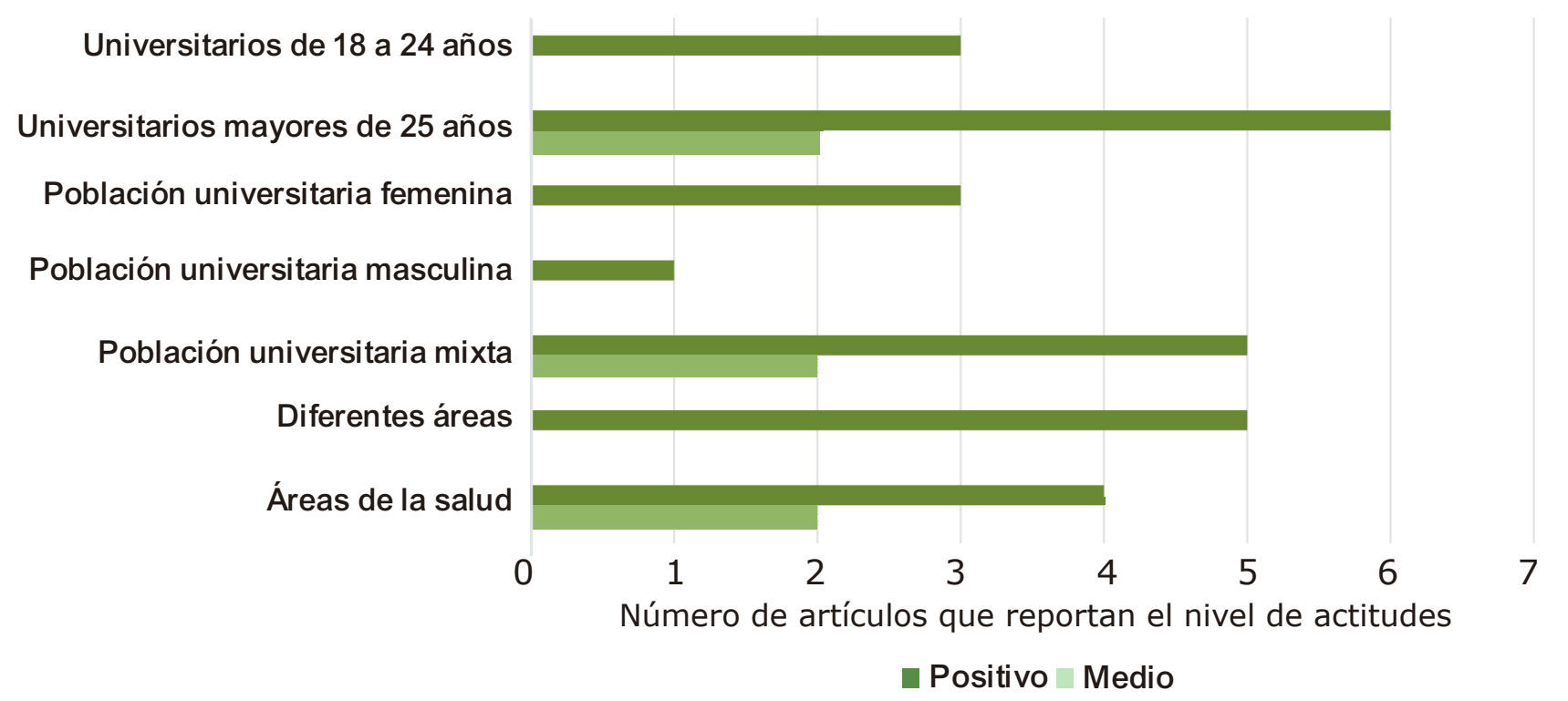

Figura 4. Nivel de conocimiento (a) y actitudes (b) por edad, sexo y área de estudio.

Figure 4. Level of knowledge (a) and attitudes (b) by age, sex and area of study.

tes varones (45\%) estuvieron de acuerdo en que la alimentación con fórmula es una mejor opción si la madre trabaja fuera del hogar en comparación con las mujeres (28\%). Al igual, Yang y col. (2019a) reportaron que el $94.3 \%$ de los estudiantes considerados en su investigación están de acuerdo en que la alimenta- ción de fórmula es la mejor opción si la madre planea salir a trabajar, ya que es más confiable, porque puede calcular la cantidad exacta de leche que está recibiendo el bebé. Sin lugar a dudas estas actitudes son resultado de las barreras de la LM, por ende, Henderson y col. (2017) en su estudio proponen la iniciati- 
va para un lugar de trabajo amigable para las madres y un clima universitario favorable para llevar a cabo la LM, así como la implementación de programas educativos que favorezcan la promoción de la LM.

\section{Lactancia materna en público}

Heathman y col. (2019), en particular, con respecto a las actitudes de la exhibición pública de la LM, fueron positivas. Los participantes estadounidenses estuvieron de acuerdo en que la exhibición pública de la LM, ya sea en una revista, cartelera o programa de televisión familiar, era igualmente aceptable. Por su parte, Lou y col. (2014) identificaron en hombres valores más altos en las actitudes respecto a la LM, en comparación a las mujeres, el $62.7 \%$ de ellos mencionó no sentirse incómodo si una mujer amamanta en público, mientras que el $13.4 \%$ de las mujeres contestó de forma contraria.

Los hombres tienden a formar actitudes favorables sobre la LM a edades tempranas (Henderson y col., 2017) y como padres, las opiniones de los hombres son muy influyentes en las elecciones de alimentación infantil de sus parejas. Los factores externos como la exposición y las actitudes mostraron una asociación más fuerte con las intenciones de amamantar, que la raza y el género (Jefferson, 2017). Sin embargo, para Jefferson (2017), los puntajes de las actitudes más altos fueron mayores para los caucásicos que para los afroamericanos, lo que demuestra una diferencia significativa por raza, aunque los puntajes de conocimiento son el único contribuyente significativo en la predicción de las intenciones de amamantar.

\section{CONCLUSIONES}

La población universitaria encuestada mostró bajo nivel de conocimiento en aspectos relevantes, tales como, la duración de la lactancia materna exclusiva, el efecto benéfico de la misma en la reducción del riesgo de desarrollo de obesidad, infecciones de oído, diarrea o alergias alimentarias. Esto fue particularmente notorio en estudiantes que no eran del área de la salud. En cuanto a las actitudes, se identificó una alta aceptación social de la práctica de la lactancia materna, pero, los estudiantes consideraron a la alimentación con fórmula como una opción más práctica. Es necesario, continuar con investigaciones que integren la evaluación conductual de los jóvenes universitarios, así como de diversos sectores de la sociedad, que permitan explicar los cambios en acciones y comportamientos hacia la lactancia materna.

\section{REFERENCIAS}

AEPED, Asociación Española de Pediatría (2012). Recomendaciones sobre lactancia materna España, en AEPED. publications. [En línea]. Disponible en: https://www.aeped.es/ sites/default/files/201202-recomendaciones-lactancia-materna.pdf. Fecha de consulta: 18 de noviembre de 2020.

González, T. y Ferré, I. (2016). Lactancia materna y beneficios para el niño, en Lactancia Materna en México. [En línea]. Disponible en: https://www.anmm.org.mx/publicaciones/ultimas_publicaciones/LACTANCIA_MATERNA.pdf. Fecha de consulta: 21 de diciembre de 2020.

Hamade, H., Farah, N., Keyrouz, S., Hwalla, N., Karam, J., Al-Rustom, L., and Nasreddine, L. (2014). Breastfeeding knowledge, attitude, perceived behavior, and intention among female undergraduate university students in the Middle East: the case of Lebanon and Syria. Food and Nutrition Bulletin. 35(2): 179-90.

Heathman, L., Douglas, C. C., and Camel, S. P. (2019).
Relationship among breastfeeding exposure, knowledge, and attitudes in collegiate males residing in East Texas. Journal of Human Lactation. 35(4): 782-789.

Henderson, J., Chapman, J., Thomas-Jackson, S., Kelly, L., and Mulsow, M. (2017). Campus Climate. Clinical Lactation. 8(4): 158-168.

Jefferson, U. T. (2017). Breastfeeding Exposure, Attitudes, and Intentions of African American and Caucasian College Students. Journal of human lactation: Official Journal of International Lactation Consultant Association. 33(1): 149-156.

Kavanagh, K. F., Lou, Z., Nicklas, J. C., Habibi, M. F., and Murphy, L. T. (2012). Breastfeeding knowledge, attitudes, prior exposure, and intent among undergraduate students. Journal of Human Lactation. 28(4): 556-564.

Linares, A. M., Parente, A. D. N., and Coleman, C. (2018). Attitudes, Practices, and Knowledge About Human Lactation Among Nursing Students. Clinic Lactation. 9(2): 59-65. 
Lou, Z., Zeng, G., Orme, J., Huang, L., Liu, F., Pang, X., and Kavanagh, K. (2014). Breastfeeding Knowledge, Attitudes, and Intention in a Sample of Undergraduate Students in Mainland China. Journal Of Human Lactation. 30(3): 331-339.

Martínez, U., Rangel, Y. y Rodríguez, E. (2017). ¿Lactancia materna o en pareja? Un estudio sobre las experiencias de reconfiguración de cuerpos, roles y cotidianeidades en madres y padres mexicanos. Cadernos de Saúde Pública. 33(9): 1-10.

Maslow, A. (1975). Motivación y personalidad (Primera edición). Barcelona: Ed. Sagitario. $145 \mathrm{Pp}$.

Mohamad, N., Saddki, N., Azman, K. N. K., and Ab-Aziz, I. D. (2019). Knowledge, Attitude, Exposure, and Future Intentions toward Exclusive Breastfeeding among University Sains Malaysia Final Year Medical and Dental Students. Korean Journal of Family Medicine. 40(4): 261.

Mosca, F. and Giannì, M. L. (2017). Human milk: composition and health benefits. Revista La Pediatria Medica e Chirurgica. (39): 47-52.

Natan, M. B., Haikin, T., and Wiesel, R. (2018). Breastfeeding knowledge, attitudes, intentions, and perception of support from educational institutions among nursing students and students from other faculties: a descriptive cross-sectional study. Nurse Education Today. (68): 66-70.

OMS, Organización Mundial de la Salud (1981). Informe sobre el estudio en colaboración de la OMS acerca de la lactancia natural Ginebra, Suiza, en WHO publications. [En línea]. Disponible en: https://apps.who.int/iris/bitstream /handle/10665/40109/9243560670.pdf?sequence=1\&isAllo wed=y. Fecha de consulta: 21 de diciembre de 2020.

OMS, Organización Mundial de la Salud (2020). Lactancia materna, en WHO publications. [En línea]. Disponible: https:// www.who.int/topics/breastfeeding/es/. Fecha de consulta: 21 de diciembre de 2020.

Paredes-Juárez, E. (2019). Conocimiento y práctica sobre lactancia materna de mujeres primigestas derechohabientes de una Unidad de Medicina Familiar. Unidad de Medicina Familiar. Revista de Enfermería del Instituto Mexicano del Seguro Social.26(4): 239-247.

Patil, D., Pundir, P., Dhyani, V., Krishnan, J., Parsekar, S., D’Souza, S., ..., and Renjith, V. A. (2020). Mixed-methods systematic review on barriers to exclusive breastfeeding. Nutrition and Health. 26(4): 323-346.

PEDro, Physiotherapy Evidence Database (2012). Estadisticas de PEDro 2012. [En línea]. Disponible en: https://pedro.org.au/spanish/learn/pedro-statistics/\#: :text=La\%20 escala\%20PEDro\%20fue\%20desarrollada,va\%20de\%200\%20 a\%2010. Fecha de consulta: 10 de diciembre de 2020.

Seidel, A. K., Schetzina, K. E., Freeman, S. C., Coulter, M.
M., and Colgrove, N. J. (2013). Comparison of breast-feeding knowledge, attitudes, and beliefs before and after educational intervention for rural Appalachian high school students. Southern Medical Journal. 106(3): 224-229.

Vandewark, A. (2014). Breastfeeding Attitudes and Knowledge in Bachelor of Science in Nursing Candidates. The Journal of Perinatal Education. 23(3):135-141.

Yang, S., Burns, E., Salamonson, Y., and Schmied, V. (2019b). Expectations and experiences of nursing students in supporting new mothers to breastfeed: a descriptive qualitative study. Journal of Clinical Nursing. 28(11-12):2340-2350.

Yang, S., Schmied, V., Burns, E., and Salamonson, Y., (2019a). Breastfeeding knowledge and attitudes of baccalaureate nursing students in Taiwan: A cohort study. Women and Birth. 32(3): 334-340. 